\title{
O PAPEL DA RADIOLOGIA NA UNIDADE DE TERAPIA INTENSIVA
}

\author{
THE ROLE OF RADIOLOGY IN INTENSIVE CARE UNITS
}

Fabiano R. Lucchesi' : Gilberto Taketani': Jorge Elias Jr² \& Clóvis S. Trad²

${ }^{1}$ Médico Residente. ${ }^{2}$ Docente do Centro de Ciências das Imagem e Física Médica. Hospital das Clínicas da Faculdade de Medicina de Ribeirão Preto.

CorResPondência: Av. Bandeirantes, 3900 - Campus Universitário, Ribeirão Preto-São Paulo. Email: cstrad@fmrp.usp.br.

LUCCHESI FR et al. O papel da radiologia na Unidade de Terapia Intensiva. Medicina, Ribeirão Preto, 31: 517-531, out./dez. 1998.

RESUMO: O presente trabalho tem o intuito de abranger da forma mais ampla possível, os diferentes meios diagnósticos disponíveis atualmente para a avaliação de pacientes em Unidade de Terapia Intensiva ( UTI ). Desta forma, alguns conceitos radiológicos essenciais para a interpretação e correta solicitação de exames serão abordados com um enfoque prático e objetivo.

UNITERMOS: Radiologia. Radiografia Torácica. Radiografia Abdominal. Unidades de Terapia Intensiva.

\section{INTRODUÇÃ̃}

A crescente incorporação da tecnologia nos diversos segmentos da medicina, particularmente na radiologia, vem contribuindo para um diagnóstico cada vez mais precoce e acurado das diferentes patologias, trazendo imensuráveis benefícios aos pacientes. Certamente, em Unidades de Terapia Intensiva (UTIs), a precocidade no diagnóstico e na detecção de possíveis complicações influencia, decisivamente, a conduta terapêutica e, em última análise, o prognóstico do paciente. O presente artigo abordará o atual papel dos diferentes métodos de diagnóstico por imagem nas patologias mais freqüentes em UTIs; e, em virtude da abrangência do assunto, descreveremos, oportunamente, os aspectos radiológicos em UTIs pediátricas e aqueles ligados ao trauma. Avaliaremos, seqüencialmente, o aparelho cardiovascular pulmonar e digestivo, enfocando, principalmente, as apresentações radiológicas típicas, sem descrições pormenorizadas dos aspectos clínicos, os quais serão analisados nos demais capítulos.

\section{APARELHOS CARDIOVASCULAR E PULMONAR}

A análise radiológica do aparelho cardiovascular basicamente é feita através da radiografia de tórax no leito, com aparelho portátil, em incidência anteroposterior e com o paciente em decúbito dorsal. Desta forma, a qualidade da imagem é inferior em relação àquela obtida no departamento de radiologia, em virtude da menor distância foco filme e posição supina, das características técnicas dos aparelhos móveis, da não utilização de grades de filtragem e, também, de possíveis imperfeições no processo de revelação do filme. Eventualmente, para melhor elucidação diagnóstica, utilizam-se incidências laterais. A radiografia digital, de utilização mais recente, possibilita, pela conversão de imagens analógicas em digitais, que estas sejam armazenadas e posteriormente manipuladas, melhorando suas características diagnósticas, tais como grau de exposição, brilho e contraste, reduzindo a repetição de exames e irradiação desnecessária dos pacientes. Também permite a reprodu- 
ção das grafias sempre que desejável e, principalmente, a transmissão, à distância, das informações, tecnologia esta conhecida como telerradiologia, com acesso rápido das imagens e laudos por todo o ambiente hospitalar, em especial as UTIs ${ }^{(1,2)}$. (Tabela I)

\section{Tabela I - Radiologia Convencional X Digital em UTI}

Desvantagens da Radiologia Convencional em UTI

- Posição supina e menor distância foco filme $\rightarrow$ ampliação do mediastino e coração, menores volumes pulmonares e análise prejudicada da trama pulmonar.

- Uso de aparelhos móveis $\rightarrow$ exposições prolongadas, possibilitando artefatos de movimento.

- Não utilização de grades de filtragem $\rightarrow$ aumento da radiação espalhada.

- Erros no processo de revelação $\rightarrow$ degradação da qualidade final da imagem.

Vantagens da Radiologia Digital em UTI

- Redução significativa da repetição de exames, diminuindo exposição à radiação.

- Possibilidade de pós-processamento das imagens em diferentes formatos, melhorando a qualidade das imagens e capacidade diagnóstica.

- Capacidade de armazenamento e transmissão das imagens. sentação radiológica é variável, desde uma radiografia normal, ou com os clássicos sinais de desvio ipsilateral das cissuras, do mediastino, dos arcos costais e das estruturas broncovasculares e elevação do hemidiafragma. Outros sinais indicativos de atelectasia são a presença de velamentos lineares ou discóides, associados ou não a broncograma aéreo ou até consolidação lobar. Quando a atelectasia cursa com ausência de broncograma aéreo, sugere a presença de obstrução da luz brônquica, possivelmente secundária à rolha de secreção, orientando, desta forma, a conduta terapêutica ${ }^{(3,4,5)}$. (Figura 1)

\subsection{Pneumonia}

A pneumonia hospitalar apresenta uma incidência em torno de $8 \%$ a $12 \%$ em UTIs clínicas e cirúrgicas, atingindo uma taxa de até $60 \%$ nos pacientes com síndrome da angústia respiratória do adulto (SARA) e uma mortalidade variando entre $13 \%$ a $55 \%$. Em ambientes de UTIs, predominam, como agentes etiológi$\mathrm{cos}$, as bactérias aeróbicas gram-negativas (Pseudomonas, Enterobacter, Klebsiella, Proteus, Acinetobacter, Serratia, Haemophilus), o Staphylococcus aureus e a Candida. Na grande maioria das vezes, há uma prévia colonização da orofaringe, bem como do estômago, principalmente quando existirem níveis

\subsection{Atelectasia}

Condição muito freqüente em UTI, resultante, geralmente, de hipoventilação pulmonar, anestesias prolongadas, posição supina, secreções pulmonares, pós-operatório e do mal posicionamento de cânulas endotraqueais. Acomete, preferencialmente, o lobo inferior esquerdo (66\%), seguido dos lobos inferior (22\%) e superior (11\%) direitos. O diagnóstico radiológico diferencial deve ser feito com outras causas de consolidação pulmonar, como pneumonia, hemorragia, infarto pulmonar, devendo-se valorizar a evolução temporal dos achados, uma vez que as mudanças na atelectasia ocorrem em horas e as demais, em dias. A apre-

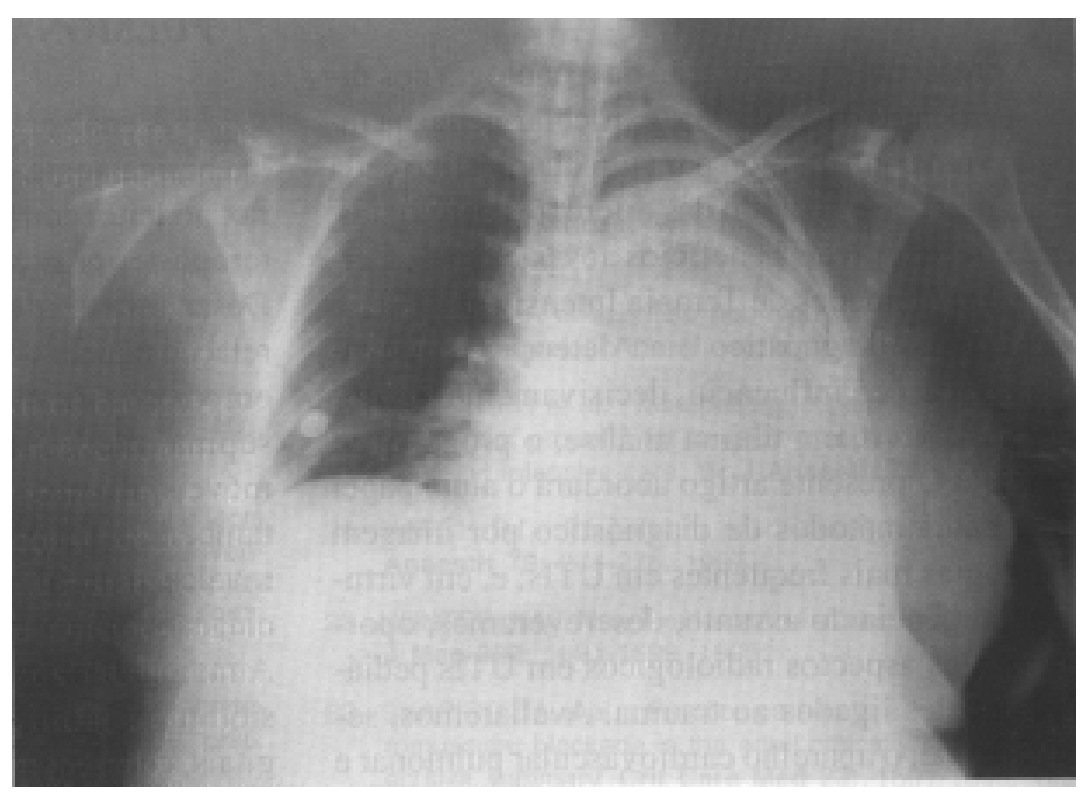

Figura 1 - Extremidade distal da cânula orotraqueal ao nível do brônquio fonte direito, associado a opacificação do pulmão esquerdo, compatível com atelectasia. 
mais elevados de $\mathrm{pH}$ gástrico, antes do desenvolvimento do quadro pneumônico. Os parâmetros utilizados para o diagnóstico da pneumonia são a presença de febre, leucocitose, leucopenia, isolamento microbiológico do agente em secreção traqueal e velamento na radiografia após setenta e duas (72) horas de internação. É importante ressaltar que estes critérios não são específicos da pneumonia, podendo ocorrer em outras condições clínicas. O diagnóstico radiológico baseia-se no aparecimento de velamento segmentar lobar ou difuso, do tipo alveolar, ou seja, com bordas mal definidas, com broncograma aéreo e sinal da silhueta $^{(3,4,5)}$ (Figura 2, Tabela II). O

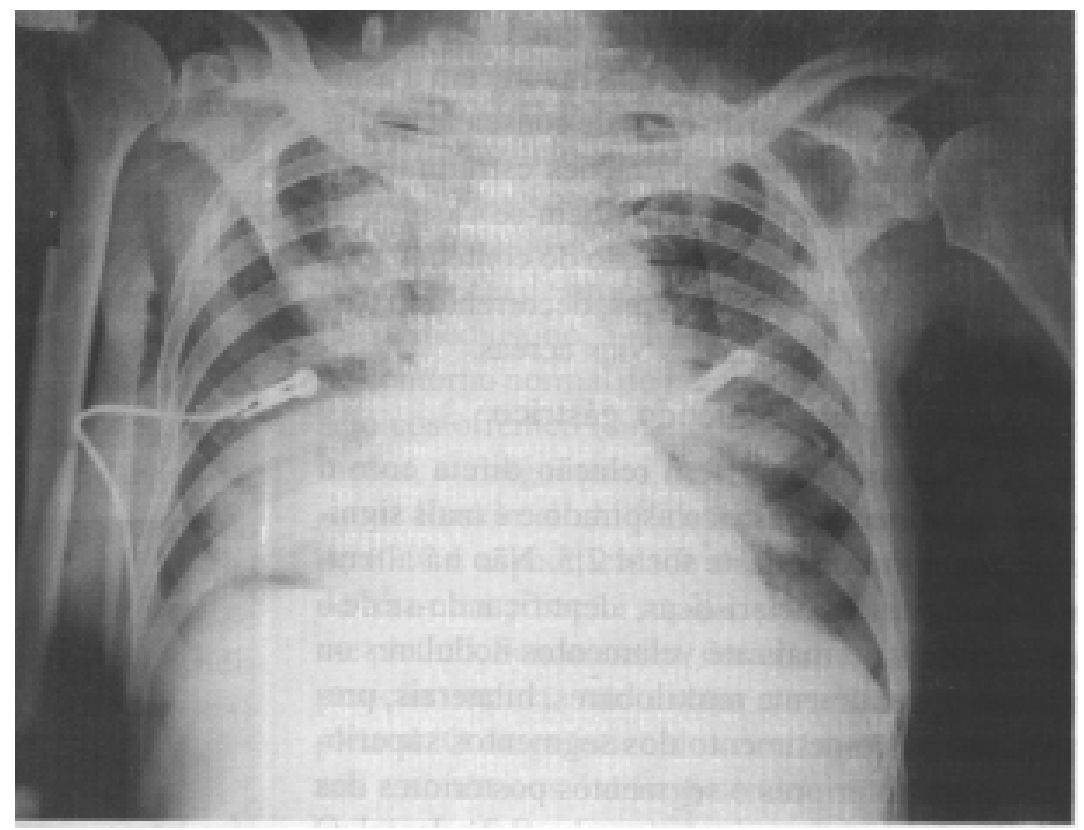

Figura 2 - Velamento de padrão alveolar, na base pulmonar direita, e infrailar esquerdo. Pneumonia bilateral.

diagnóstico diferencial deve

ser feito com atelectasia, hemorragia, infarto, contusão, derrame pleural, edema e SARA, sendo que esta última patologia dificulta sobremaneira o diagnóstico de pneumonia ${ }^{(6)}$ (Tabela III).

Infelizmente, a especificidade da radiografia de tórax para detecção de pneumonia é baixa, variando de $27 \%$ a $35 \%$, mesmo considerando-se os dados clínicos e as radiografias anteriores. A presença de cavitação é sugestiva de pneumonia. O velamento devido ao processo pneumônico costuma aparecer mais tardiamente e resolver-se mais lentamente que a aspiração pulmonar e a atelectasia. Um velamento que tenha surgido em horas é sugestivo de atelectasia, enquanto o aparecimento súbito de qualquer novo velamento deve levantar a suspeita de aspiração.

Em geral, mesmo com adequada terapêutica antibiótica, o padrão radiológico não se altera de forma importante em quarenta e oito (48) horas, sendo considerado tratamento ineficiente, quando não houver melhora radiológica em quatorze (14) dias. As mudanças das condições clínicas, geralmente, precedem as alterações radiológicas ${ }^{(7,8)}$. A tomografia computadorizada, com sua capacidade de detectar alterações no parênquima pulmonar, derrame pleural, bem como alterações nas demais estruturas torácicas, tem papel coadjuvante à radiografia de tórax na detecção
Tabela II - Características Radiológicas do Velamento de padrão alveolar

- Margens mal definidas

- Distribuição lobar ou segmentar

- Broncograma aéreo

- Coalescente

- Tempo de resolução rápido

Tabela III - Diagnóstico diferencial e radiológico de pneumonia

$\begin{array}{ll}\text { Contusão } & \text { TEP } \\ \text { Atelectasia } & \text { Hemorragia } \\ \text { Infarto pulmonar } & \text { Derrame pleural } \\ \text { Edema } & \text { SARA }\end{array}$

de pneumonia e suas eventuais complicações ${ }^{(9,10)}$. Entretanto, nem sempre é possível sua utilização em pacientes mantidos em UTIs, em função do estado clínico deteriorado do enfermo, apesar da melhora atual da segurança no transporte de pacientes graves. 


\subsection{Pneumonia Aspirativa}

A ocorrência de aspiração em UTIs é bastante freqüiente e as condições que favorecem a aspiração são o rebaixamento do nível de consciência, distúrbios neuromusculares e alterações estruturais no tubo digestivo. Classicamente, dividem-se a aspiração e suas conseqüências em: aspiração do conteúdo gástrico, infecções pleuropulmonares, decorrentes da aspiração e obstrução aguda das vias aéreas.

\subsubsection{Aspiração do conteúdo gástrico}

A lesão pulmonar tem relação direta com o volume do conteúdo gástrico aspirado e é mais significativa quando o $\mathrm{pH}$ deste for $<2,5$. Não há alterações radiológicas características, identificando-se desde radiografias normais até velamentos nodulares ou confluentes, geralmente multilobares, bilaterais, predominando o acometimento dos segmentos superiores dos lobos inferiores e segmentos posteriores dos lobos superiores, em pacientes em decúbito dorsal. O aspirado gástrico também poderá desencadear edema pulmonar não cardiogênico.

\subsubsection{Complicações infecciosas}

Na pneumonia aspirativa comunitária há um predomínio das bactérias anaeróbicas como agentes etiológicos (Bacteriodes fragilis, Fusobacterium nucleatum, Peptostreptococcus spp) e das bactérias aeróbicas nas pneumonias nosocomiais, refletindo a colonização da orofaringe (Haemophilus influenzae, Staphylococcus aureus, bacilos gram-negativos).

\subsubsection{Obstrução das vias aéreas}

As alterações radiológicas dependerão do nível da obstrução na árvore traqueobrônquica, podendo ser normal ou mostrar hiperinsuflação unilateral, atelectasia e desvio do mediastino ${ }^{(11,12,13)}$.

\subsection{Edema Pulmonar}

Havendo um aumento da pressão hidrostática ao nível dos capilares pulmonares, decorrente do estado de hiperhidratação ou do aumento da pressão diastólica final do ventrículo esquerdo $\left(\mathrm{Pd}_{2} \mathrm{VE}\right)$, ocorrerá um extravasamento de líquido, inicialmente, para o interstício e, depois, para o alvéolo. Como os pacientes em UTIs estão geralmente em posição supina, a redistribuição da trama vascular pulmonar, resultante do aumento da $\mathrm{Pd}_{2} \mathrm{VE}$ não é identificada. As alterações radiológicas do edema intersticial compreendem as linhas septais de Kerley e borramento da trama vascular pulmonar. Com o extravasamento de líquido atingindo os alvéolos, nota-se velamento do tipo flocoso, coalescente, de limites mal definidos, periilar, com aspecto em "asa de borboleta" e presença de broncograma aéreo. Geralmente, é bilateral e simétrico, associado a área cardíaca aumentada, apresentando início e resolução rápida, diferenciando-o da pneumonia e da Síndrome da Angústia Respiratória do Adulto ${ }^{(3,4,5)}$. (Figura 3)

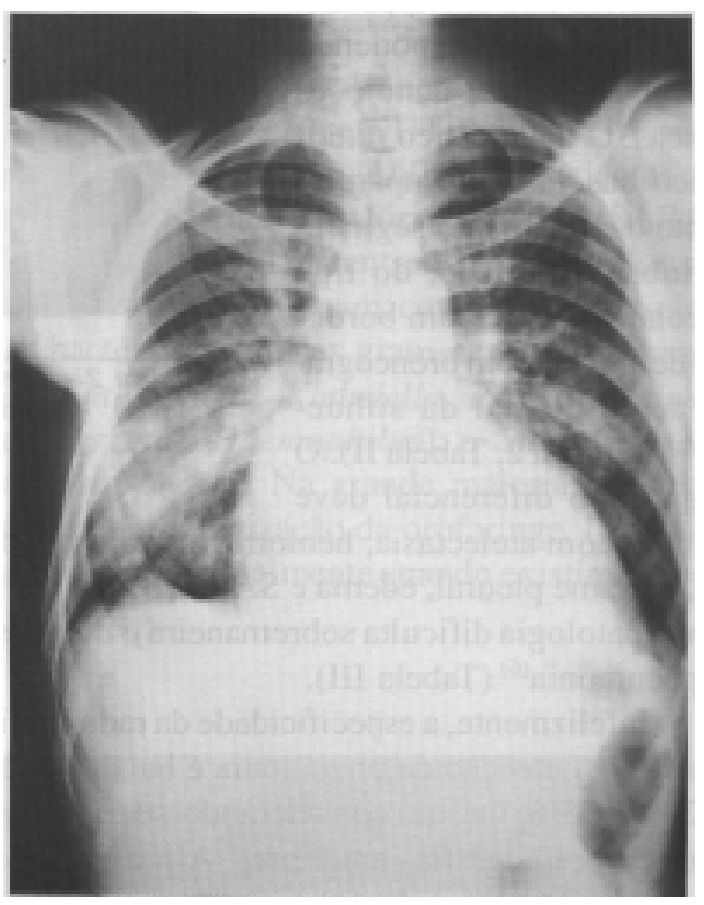

Figura 3 - Edema pulmonar cardiogênico. Aumento da área cardíaca, associado a velamento de padrão radiológico alveolar, com distribuição periilar, mais acentuado à direita.

\subsection{Síndrome da Angústia Respiratória do Adulto}

O diagnóstico da Síndrome da Angústia Respiratória do Adulto (SARA) é baseado em critérios clínicos, gasométricos, hemodinâmicos e radiológicos. Apresenta-se como dispnéia pronunciada, com complacência pulmonar diminuída, hipoxemia refratária e pressão capilar pulmonar $<18 \mathrm{mmHg}$. Em termos fisiopatológicos e radiologicamente, distinguem-se três fases:

Estágio I (0 -24h) 
Trata-se da fase exsudativa inicial, com discreto extravasamento de líquido para o interstício, traduzindo-se por uma radiografia de tórax normal ou com aparecimento de linhas septais.

\section{Estágio II (24-36h)}

Nesta fase, aumenta o extravasamento de líquido para o interstício e para o alvéolo, manifestando-se por opacificação alveolar, de limites mal definidos, localizados, inicialmente, nas porções mais periféricas dos pulmões, progredindo para o envolvimento difuso dos campos pulmonares. A presença de broncograma aéreo, ausência de cardiomegalia e alargamento do pedículo vascular ajudam a diferenciá-la do edema pulmonar cardiogênico. Geralmente, após trinta e seis (36) horas, os achados radiológicos mantêm-se inalterados, sendo que qualquer mudança pode indicar processo infeccioso associado.

\section{Estágio III ( \pm 72h)}

Caracteriza-se pelo processo de reparação pulmonar, com resolução do quadro alveolar, evidenciando-se velamento de padrão reticular.

Dentre as complicações mais freqüentemente associadas estão a pneumonia e o barotrauma, este último podendo se manifestar sob a forma de enfisema intersticial, enfisema subcutâneo, cistos subpleurais, pneumotórax, pneumomediastino, pneumoperitôneo e pneumorretroperitôneo ${ }^{(14)}$.

A tomografia computadorizada de tórax é importante na identificação de complicações não diagnosticadas na radiografia convencional, especialmente abscessos, enfisemas, doenças mediastinais e na quantificação do acometimento pulmonar ${ }^{(9,10)}$. (Tabela IV)

\subsection{Derrame Pleural}

Na posição em decúbito dorsal, em função da gravidade, o líquido ficará localizado, preferencialmente, nas porções posteriores do ápice e base do hemitórax, traduzindo-se, radiologicamente, como um aumento homogêneo da densidade do hemitórax envolvido, mantendo-se a visualização dos vasos pulmonares, ausência de broncogramas aéreos, sem desvio do hilo e mediastino, a não ser quando volumoso, perda do contorno normal do hemidiafragma, obliteração do seio costofrênico (sinal do menisco) e do ápice pulmonar, e alargamento da cissura menor ${ }^{(3,4,5,15)}$.

Ruskin et al. ${ }^{(16)}$ descrevem uma sensibilidade de $67 \%$ e especificidade de $70 \%$ para a radiografia de tórax na posição supina, para detecção de derrame pleural. Sendo assim, uma radiografia de tórax, na posição supina, não exclui a presença de derrame pleural. O diagnóstico dos derrames pleurais pequenos e simétricos é difícil, sendo importante a contribuição do ultra-som e da tomografia computadorizada. Woodring e Collins et al. ${ }^{(17,18)}$ relatam que, provavelmente, um derrame pleural não seja diagnosticado nas grafias em posição supina, quando tiver um volume menor do que $175 \mathrm{ml}$. A radiografia de tórax não tem a capacidade de distinguir entre exsudato, transudato, empiema e hemorragia, o que pode ser melhor avaliado através da tomografia computadorizada. $\mathrm{O}$ derrame pleural poderá estar loculado, geralmente correspondendo a um exsudato (neoplasia, sangue ou empiema), não se alterando nas grafias, em diferentes projeções. O diagnóstico diferencial deverá ser feito entre atelectasia e outros processos pulmonares que promovam consolidação.

\begin{tabular}{||lccc|}
\hline \multicolumn{2}{|l|}{ Tabela IV - Características radiológicas das principais causas de edema pulmonar } \\
\hline & Cardiogênico & Renal & $\begin{array}{c}\text { Síndrome Angústia } \\
\text { Respiratória Adulto }\end{array}$ \\
\hline Área cardíaca & Aumentada & Aumentada & Normal \\
Derrame pleural & Comum & Comum & Incomum \\
Broncograma áerea & Incomum & Incomum & Comum \\
Distribuição do edema & Central/periférico & Central/periférico & Periférico \\
Distribuição do fluxo sanguíneo & Invertido & Balanceado & Normal ou balanceado \\
Pedículo vascular* & Normal ou aumentado & Aumentado & Normal ou reduzido
\end{tabular}

*Avaliação prejudicada em decúbito dorsal. 


\subsection{Pneumotórax}

Trata-se de uma das entidades mais freqüentemente encontradas em UTIs, seja em virtude da larga utilização da ventilação mecânica, ou devido a procedimentos diagnósticos ou terapêuticos ou resultantes das patologias presentes em pacientes gravemente enfermos.

Estima-se que a incidência de pneumotórax, em pacientes sob ventilação mecânica, varie entre $4 \%$ a $15 \%$, sendo que cerca de $60 \%$ a $96 \%$ podem, rapidamente, tornar-se hipertensivos. Radiologicamente, o pneumotórax é identificado como separação das pleuras parietal e visceral, associada a ausência de vasculatura entre os folhetos pleurais, sendo melhor visualizado na grafia em expiração. Em função da posição supina e da gravidade, $o$ ar, preferencialmente, coletase nas regiões anteromedial e subpulmonar e, quando mais volumoso, também na região látero-apical. Identifica-se a localização anteromedial pelo adequado delineamento das estruturas mediastinais, tais como as veias cava superior e ázigo, veias pulmonares superiores, artéria subclávia esquerda, veia cava inferior e contornos cardíacos. Observa-se, na localização subpulmonar, aumento da radiotransparência dos quadrantes superiores do abdome, seio costofrênico mais profundo e hipertransparente, adequada visualização do diafragma ipsilateral e margem inferior do pulmão. Em presença de perda de volume do lobo inferior, o ar poderá coletar-se na região posteromedial, delineando as estruturas do mediastino posterior, como a aorta descendente, seios costovertebrais e linha paraespinhal $^{(19 / 24)}$.

Tanto a perda de volume pulmonar quanto o desvio do mediastino podem ocorrer no pneumotórax simples e no hipertensivo, sendo que, neste último, notam-se as repercussões clínicas dos distúrbios respiratórios e hemodinâmicos. Radiologicamente, identifica-se deslocamento inferior ou inversão do diafragma e alteração do contorno do coração e dos vasos.

Pneumotórax de pequeno volume pode passar despercebido à radiologia convencional, sendo diagnosticado na tomografia computadorizada.

\subsection{Tromboembolismo Pulmonar}

O tromboembolismo pulmonar (TEP) é uma condição bastante freqüente num ambiente de UTI, uma vez que estes pacientes possuem vários fatores de risco com relação à clássica tríade de Virchow (injúria vascular, estase sangüínea e estados de hi- percoagulabilidade). Infelizmente, tanto os dados clínicos quanto os radiológicos são bastante inespecíficos e de baixa sensibilidade. A radiografia de tórax tem importância no sentido de identificar eventuais diagnósticos diferenciais, a saber, pneumonia, pneumotórax, edema pulmonar. Dentre os achados radiográficos estão o derrame pleural $(50,9 \%)$, diminuição focal da trama vascular pulmonar (Sinal de Westermark - 15\%), elevação do diafragma, atelectasia, dilatação da artéria pulmonar e consolidação. Cerca de $10 \%$ a $15 \%$ dos TEP promovem infarto pulmonar, sendo mais comuns em lobos inferiores, apresentando-se como opacificação adjacente a pleura, ocasionalmente no formato piramidal, com ponta para o hilo (Sinal de Hampton), aparecendo entre doze (12) a vinte e quatro (24) horas do início dos sintomas. (Figura 4). Num período de três (03) semanas, pode resolver-se completamente ou deixar espessamento pleural e traves fibróticas ${ }^{(4,5,25)}$.

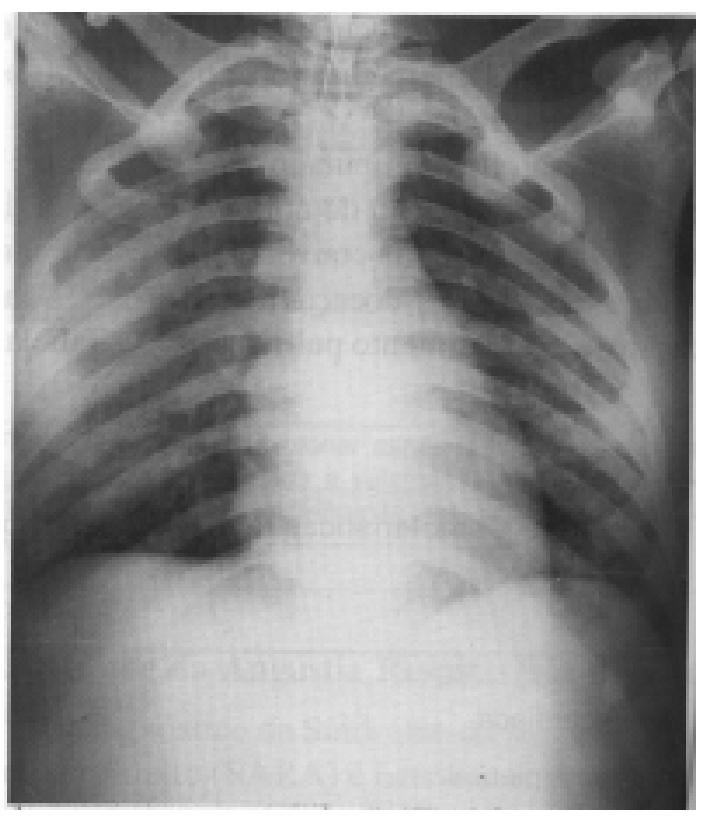

Figura 4 - Tromboembolismo pulmonar, confirmado pelo estudo de ventilação e perfusão. Velamento alveolar com base voltada para margem lateral do gradeado costal direito. 
Outras modalidades diagnósticas são ventilação e perfusão com material radioativo, angiografia pulmonar, tomografia computadorizada espiral e, mais recentemente, a ressonância nuclear magnética. Os resultados do estudo com ventilação/perfusão devem ser correlacionados com os dados clínicos dos pacientes, uma vez que um resultado anormal não necessariamente confirma a suspeita clínica de tromboembolismo pulmonar.

\subsection{Dispositivos}

\subsubsection{Sonda Endotraqueal}

Relata-se que, em cerca de $12 \%$ a $15 \%$ dos pacientes, há um mau posicionamento da sonda endotraqueal, sendo este detectado, ao exame físico, em apenas 3\% dos casos. A posição ideal da extremidade distal da sonda com a cabeça em posição neutra é de 5 a $7 \mathrm{~cm}$ acima da carina, já que a sonda poderá migrar cerca de $2 \mathrm{~cm}$ para cima ou para baixo, com o movimento de flexão e de extensão da cabeça. Quando houver dificuldade em localizar a carina na radiografia de leito, é prudente deixar a extremidade distal do tubo ao nível do corpo vertebral T2 - T4, uma vez que, em 90\% dos casos, a carina está entre T5 e T7, na radiografia de leito. Em função da angulação do brônquio fonte direito, este freqüentemente é entubado seletivamente, promovendo hiperinsuflação do pulmão direito e atelectasia do esquerdo. Além da entubação seletiva, eventualmente, poderá ocorrer entubação do esôfago. Naqueles pacientes onde se prolonga o período de ventilação mecânica, indica-se a traqueostomia, sendo que a cânula deverá ser colocada ao nível de T3, a cerca de 2/3 da distância entre o estoma traqueal e a carina. Apresentam-se, como possíveis complicações, o pneumotórax, pneumomediastino, enfisema subcutâneo, hemorragia, mau posicionamento, estenose traqueal, fístula traqueoesofágica e infecção da ferida.

\subsubsection{Cateter venoso central}

A extremidade distal do cateter deverá estar localizada entre o átrio direito e as valvas venosas mais proximais, que estão localizadas nas veias subclávias e na jugular interna, cerca de $2,5 \mathrm{~cm}$ do ponto onde estes vasos se juntam para formar a veia braquiocefálica. A última valva na veia subclávia está situada ao nível do primeiro arco costal anterior. Assim, a ponta do cateter deverá estar localizada medialmente à porção anterior do primeiro arco costal. Não se deve deixar a ponta do cateter no átrio ou ventrículo direito, em função da possibilidade de arritmias, perfuração e tamponamento cardíaco. Outras complicações são o pneumotórax (1\% a 12\%), hemotórax, hemorragia mediastinal, embolia gasosa e fratura do cateter.

\subsubsection{Sonda nasogástrica}

A ponta deve estar localizada cerca de $10 \mathrm{~cm}$ além da junção gastroesofágica, para que o último orifício lateral fique localizado dentro do estômago, prevenindo a infusão da dieta no esôfago e posterior aspiração pulmonar. A correta verificação do posicionamento da sonda é importante para excluir a eventual colocação em vias aéreas e conseqüente infusão da dieta na árvore traqueobrônquica.

\subsubsection{Cateter de Swan Ganz}

A ponta do cateter não deve estar além da porção proximal das artérias pulmonares interlobares, pois, quando localizada mais distalmente, aumenta a chance de infarto pulmonar. As possíveis complicações que devem ser investigadas radiologicamente incluem o infarto pulmonar, perfuração, pneumotórax, enovelamento do cateter, mal posicionamento, ruptura da artéria pulmonar. (Figura 5)

\subsubsection{Balão intra aórtico}

O correto posicionamento da ponta do balão deve ser distal à origem da artéria subclávia esquerda, ao nível do botão aórtico, evitando oclusão das artérias carótidas, subclávias e renais. As complicações incluem embolia cerebral, dissecção aórtica, oclusão de vasos emergentes da aorta e ruptura do balão.

\subsubsection{Marcapasso}

A ponta do fio de marcapasso deve localizarse na ponta do ventrículo direito, pelo menos $3 \mathrm{~mm}$ aprofundada na gordura epicárdica, na grafia em perfil. A importância da radiologia é na detecção de complicações, como mau posicionamento, fratura do fio, perfuração miocárdica e tamponamento cardíaco.

\subsubsection{Dreno torácico}

O estudo radiológico é importante para determinação do posicionamento, sendo também necessário à obtenção de grafia em perfil, para determinar se os tubos de drenagem estão bem localizados em relação à coleção. Quando houver loculação, a drenagem também poderá ser guiada pelo ultra-som ou tomografia computadorizada. As complicações mais importantes são o inadequado posicionamento, perfuração pulmonar e fístula broncopleural ${ }^{(4,5,26)}$. 


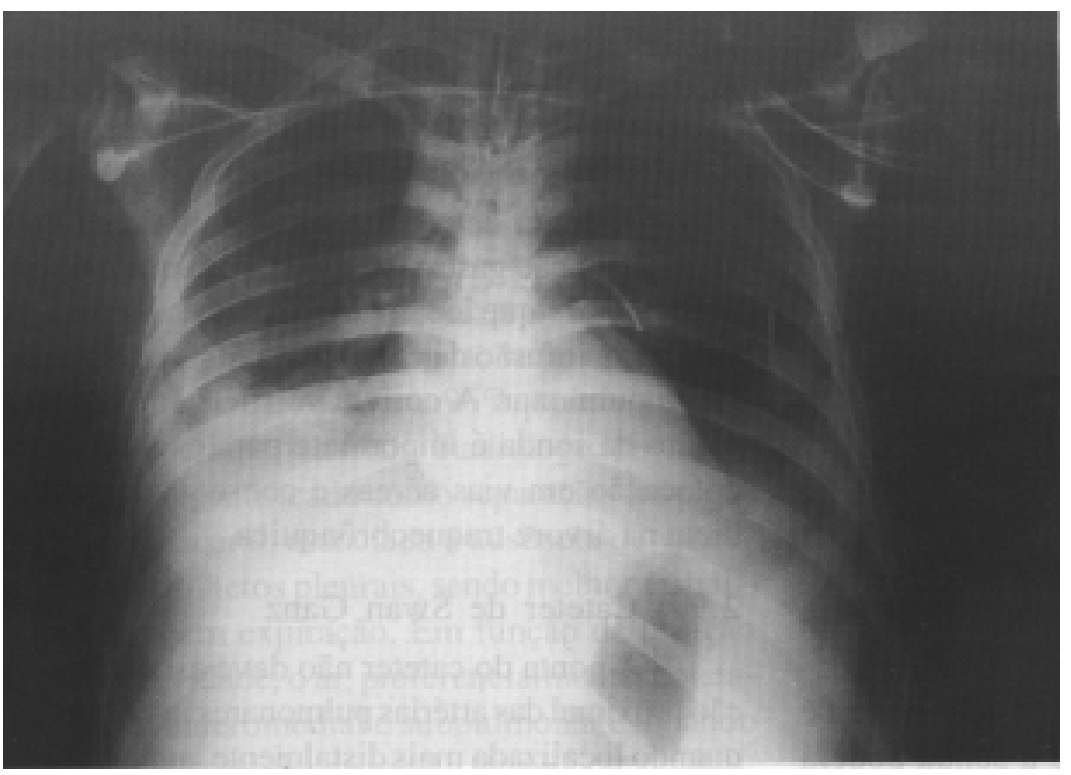

Figura 5 - Rx de tórax, revelando adequado posicionamento do cateter de Swan Ganz, que se encontra na porção proximal da artéria interlobar esquerda.

\subsection{Pneumoperitônio}

O acúmulo de gás, na cavidade peritoneal, é freqüentemente encontrado no pósoperatório de cirurgia abdominal recente, diálise peritoneal, laparoscopia ou outros procedimentos invasivos abdominais, além de perfuração de vísceras ocas intraperitoneais, infecção, pneumatose intestinal, e, raramente, secundário a pneumomediastino.

A duração do pneumoperitônio pós-operatório, demonstrável radiologicamente, está relacionada à quantidade de gás presente imediatamente após o procedimento responsável por este, sendo reabsorvido, em média, em quatro (4) a cinco (5) dias. Após

\section{ABDOME EM UTI}

O estudo radiológico do abdome, em ambiente de UTI, baseia-se na utilização do ultra-som, da tomografia computadorizada e da radiografia simples, sendo a seqüência de investigação empregada dependente da patologia apresentada e das limitações diagnósticas de cada método de imagem. Em virtude da gravidade clínica dos pacientes e das dificuldades de locomoção, os benefícios da tomografia computadorizada devem ser avaliados em relação aos riscos do transporte de pacientes graves até a sala de exames. Para otimização das informações fornecidas pela tomografia computadorizada dedica-se cuidadosa atenção à técnica empregada no exame, devendo o intestino delgado estar uniformemente opacificado e distendido pela administração rotineira prévia de contraste iodado oral a 2 ou 3\%, para adequada avaliação da espessura da parede intestinal, evitando-se falsas interpretações de abscessos, massa ou linfadenopatia $^{(27,28)}$.

Para pacientes gravemente debilitados, com história prévia de reação moderada ou intensa ao meio de contraste, doença cardíaca significante, asma, diabetes ou alergia a outros medicamentos, indica-se o uso de meios de contraste não iônicos, pela sua menor incidência de reações alérgicas. este período, deve-se suspeitar da possibilidade de deiscência anastomótica de vísceras ocas ou outras causas predisponentes.

Em $90 \%$ dos pacientes com pneumoperitônio espontâneo, há condição abdominal aguda que requer intervenção cirúrgica ${ }^{(29)}$.

Para a detecção de pneumoperitônio, em pacientes de UTI, freqüentemente é impossível a realização de grafias em posição ortostática, sendo necessária a avaliação em decúbito dorsal, o que permite demonstrar o pneumoperitônio em 50\% das vezes ${ }^{(30)}$. Havendo dúvida na incidência em decúbito dorsal, é possível verificar na radiografia feita em decúbito lateral esquerdo, com raios horizontais, após dez (10) minutos nesta posição, gás acumulado entre a parede abdominal e a borda lateral direita do fígado.

O sinal radiológico mais conhecido de pneumoperitônio, em grafias em decúbito, é a demonstração de gás em ambos os lados da parede intestinal (sinal de Rigler), ocasionalmente formando coleções de gás extraluminais, triangulares, entre as alças adjacentes (Figura 6). Outros sinais radiológicos são a visualização do ligamento falciforme, como uma faixa radiopaca no quadrante superior direito, quando o gás se acumula ao redor deste e a visualização das pregas umbilicais mediais, na pelve, em forma de "V" invertido. Existindo uma grande quantidade de gás, poder-se-á 


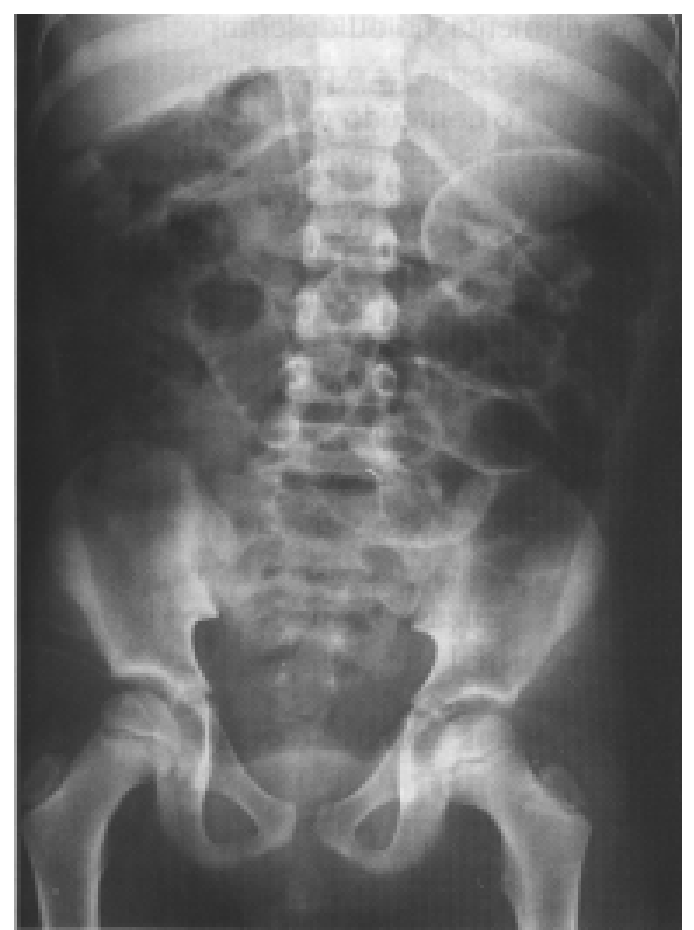

Figura 6 - Rx simples de abdome, demonstrando 0 ligamento falciforme como uma faixa radiodensa paravertebral à direita e a superfície interna e externa das alças intestinais (Sinal de Rigler)
A tomografia computadorizada é capaz de demonstrar quantidades menores de gás intraperitoneal, que se acumula sob a parede anterior do abdome, quando o paciente está em posição supina.

\subsection{Líquido livre intraperitoneal}

Na radiografia simples de abdome, podemos avaliar um grande número de sinais de líquido livre intraperitoneal, a maioria deles com uso limitado, em pacientes de UTI. Os cólons ascendente e descendente devem estar imediatamente adjacentes à faixa radiolucente da parede abdominal lateral, que constitui a gordura préperitoneal. Caso essas porções do cólon, preenchidas por gás, fezes ou contraste, estejam deslocadas medialmente, pode-se concluir, com razoável grau de confiança, que se trata de líquido livre. Outros sinais úteis são a perda da definição do ângulo hepático do cólon e área de densificação adjacente ao espaço de Douglas.

O exame ultra-sonográfico é a melhor alternativa, quando a grafia simples mostra-se inconclusiva e o paciente não pode deslocar-se até a sala de tomografia computadorizada, pois evidencia, com alta sensibilidade e especificidade, o líquido livre peritoneal, mesmo quando de pequeno volume, propiciando, muitas vezes, o diagnóstico da causa do derrame peritoneal, como, por exemplo, pancreatite, ruptura vesicular, processos inflamatórios abdominais e outros. (Tabela V) formar uma área radiolucente, ovalada, preenchendo todo o abdome, referida como "football sign", mais comumente vista em crianças e idosos. O gás pode também se acumular anteriormente, na porção central do abdome superior, freqüentemente em posição semi-ereta do paciente, dando a aparência de um "terceiro diafragma central"(31).

Mais frequiente que qualquer um desses sinais, é a demonstração de coleções gasosas anteriores ao fígado e no espaço subepático.

A demonstração de pneumoperitônio é especialmente difícil através do exame ultra-sonográfico, dependendo de sinais indiretos e de alto grau de experiência do examinador, não sendo exame indicado para tal suspeita. No entanto, pode ser importante na detecção de lesões associadas.

\begin{tabular}{|c|c|}
\hline Tipo de Líquido & Densidade tomográfica(UH) \\
\hline Transudato (ICC, Cirrose ) & 0 a 15 \\
\hline Exsudato & 15 a 30 \\
\hline Hemorrágico & $\begin{array}{l}\text { Dependente do tempo de evolução } \rightarrow 15 \text { a } 75 \\
\text { Agudamente,diminuindo com a lise do coágulo }\end{array}$ \\
\hline
\end{tabular}

Se a coleção for localizada e hiperdensa, na tomografia computadorizada, pode-se interpretar como um coágulo e, assim, predizer o local de hemorragia ("sentinel clot sign").

\subsection{Isquemia intestinal}

A isquemia intestinal é um problema relativamente comum em pacientes idosos em UTI. Sinais específicos de isquemia, na grafia simples, são vistos em menos de $20 \%$ das vezes. (Tabela VI) 
Tabela VI - Sinais radiológicos de isquemia intestinal

Radiografia simples

- Espessamento segmentar da parede intestinal e impressões digitiformes, localizadas (thumb printing).

- Gás intramural e/ou no sistema venoso portal (pneumatose).

- Gás livre intraperitoneal.

- Dilatação difusa dos intestinos delgado e grosso em $50 \%$ dos casos (padrão pseudo-obstrutivo)

Tomografia computadorizada

- Mesmos achados citados para a grafia simples.

- Espessura parietal $>3 \mathrm{~mm}$, com ou sem reforço anelar.

- Hiperdensidade parietal na fase não contrastada (usualmente $>20 \mathrm{UH}$ ) e hipodensidade e/ou heteregeneidade pós-contraste.

Liquído livre intraperitoneal.

Além desses sinais, Ha et al. ${ }^{(33)}$ demonstraram que o contorno serrilhado e o afilamento pontiagudo da luz intestinal, presentes em obstrução, sugerem a presença de estrangulamento, que tem como causas mais freqüentes aderências e hérnias.

Os distúrbios vasculares são as condições que mais freqüentemente levam à isquemia intestinal. Com o advento de uma nova técnica de TC, denominada espiral ou helicoidal, que possibilita a obtenção de cortes contíguos, em velocidade muito alta, após injeção em "bolus" de contraste, permitindo demonstrar impregnação da parede das alças, numa fase arteriolar, pode-se diagnosticar a isquemia mesentérica.

\subsection{Obstrução intestinal}

Um importante conceito, na análise da obstrução intestinal, é a compreensão de que a maior parte dos gases contidos no intestino é oriunda da deglutição, e a progressão destes depende de um trânsito intestinal livre. Desta forma, se algum segmento gastrintestinal se encontra distendido, e o adjacente não contém gás, é um bom sinal indicativo de obstrução.

Ocasionalmente, pode ocorrer o mesmo em presença de íleo adinâmico e isquemia intestinal, sendo difícil a diferenciação entre tais entidades. É útil lembrar que o íleo adinâmico, em UTI, é muito mais comum que a obstrução intestinal.

\subsection{Monitorização radiológica de tubos gastrintestinais}

A maioria dos tubos nasogástricos e intestinais, de alimentação ou descompressão, são posicionados "às cegas" e o posicionamento correto é assumido se o conteúdo gástrico puder ser aspirado ou se ar injetado puder ser ouvido na região epigástrica. Esta técnica é falível, uma vez que o barulho do gás injetado em sondas erroneamente localizadas no espaço pleural, mediastino ou pulmões pode ser ouvido no epigástrio. Assim, a checagem radiológica dos tubos e sondas deve ser rotineira, devendo - se, atentar para as possíveis complicações: hemorragias fatais por tubos pressionando grandes vasos anômalos, nós, acotovelamentos, perfuração da parede intestinal, principalmente em crianças, e aspiração e pneumonia secundárias ao mal posicionamento.

\subsection{Processos inflamatórios}

\subsubsection{Colecistite aguda}

O ultra-som continua sendo a modalidade de escolha para a detecção da colecistite aguda, embora a tomografia computadorizada possa ser útil em casos onde a visualização ultra-sonográfica da vesícula biliar é insatisfatória.

Os critérios sonográficos para o diagnóstico de colecistite aguda incluem: cálculo fixo infundibular ou obstruindo o ducto cístico, dilatação da vesícula biliar (diâmetro transverso $>40 \mathrm{~mm}$ ), espessamento da parede vesicular (> 3mm), líquidos pericolecísticos e dor à compressão do transdutor em projeção da vesícula (sinal de Murphy ecográfico), que, em pacientes inconscientes, pode ser de difícil avaliação. A colecistite enfisematosa e a perfuração são complicações da colecistite aguda, detectáveis ao US. A colecistite enfisematosa ocorre mais frequientemente em pacientes diabéticos, e o diagnóstico deve ser suspeitado quando se observam ecos reverberativos na parede e lúmen da vesícula ao US e áreas radiolucentes, na projeção vesicular ao RX. Ocorrendo perfuração vesicular, o US pode demonstrar abcesso pericolecístico.

A colecistite alitiásica é definida como inflamação da vesícula biliar na ausência de cálculos, é mais comum em pacientes em estado crítico ou em pósoperatório recente, queimados, sepse, hipotensão prolongada, diabéticos e hiperalimentação.

Os critérios diagnósticos incluem espessamento parietal, com espessura maior que $3 \mathrm{~mm}$, líquido 
pericolecístico, edema subseroso na ausência de ascite, gás intramural, distensão vesicular e bile hiperdensa pela tomografia computadorizada.

\subsubsection{Abcessos abdominais}

Podem estar localizados na parede abdominal, na cavidade peritoneal, dentro de vísceras sólidas ou no retroperitôneo. A maioria dos casos são precedidos por cirurgia abdominal, trauma, perfuração ou infecção de estruturas abdominais.

A grafia simples poderá demonstrar coleções gasosas extraluminais deslocando alças intestinais, elevação da cúpula diafragmática (abcessos subdiafragmáticos), com redução de sua excursão respiratória, mimetizando coleções líquidas subpleurais.

À ultra-sonografia, um abcesso pode assumir várias configurações, sendo mais comum uma coleção fluida sonolucente, com reforço acústico posterior, paredes espessas e irregulares, níveis líquido líquido, septos ou debris no interior e, eventualmente, reverberação, sugerindo gás. Abcessos com ecos internos difusos podem simular massas neoplásicas sólidas ${ }^{(34)}$.

Na tomografia computadorizada, os abcessos podem se apresentar como coleções com densidade de partes moles $(0$ a $40 \mathrm{UH})$ que causam efeito de massa ou se comunicam através de fístulas com estruturas adjacentes, tendo paredes espessadas e irregulares que reforçam após a administração de contraste EV. Gases e debris podem também ser identificados.
A acuracidade do método alcança $90 \%$ em algumas séries ${ }^{(35)}$, embora a distinção definitiva entre coleções estéreis e sépticas necessite de punção aspirativa, guiada, através ou do ultra-som.(Figuras 7 e 8 ).

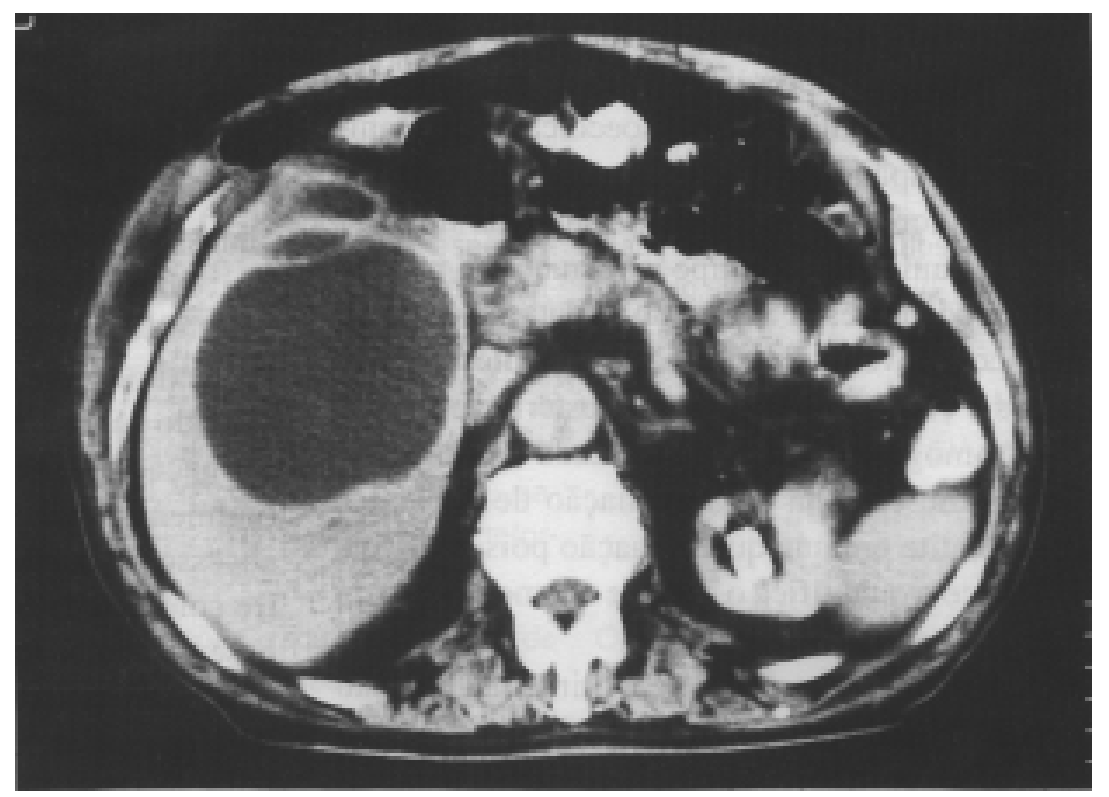

Figura 7 - Tomografia contrastada evidenciando um abcesso hipodenso, com reforço periférico no lobo hepático direito.

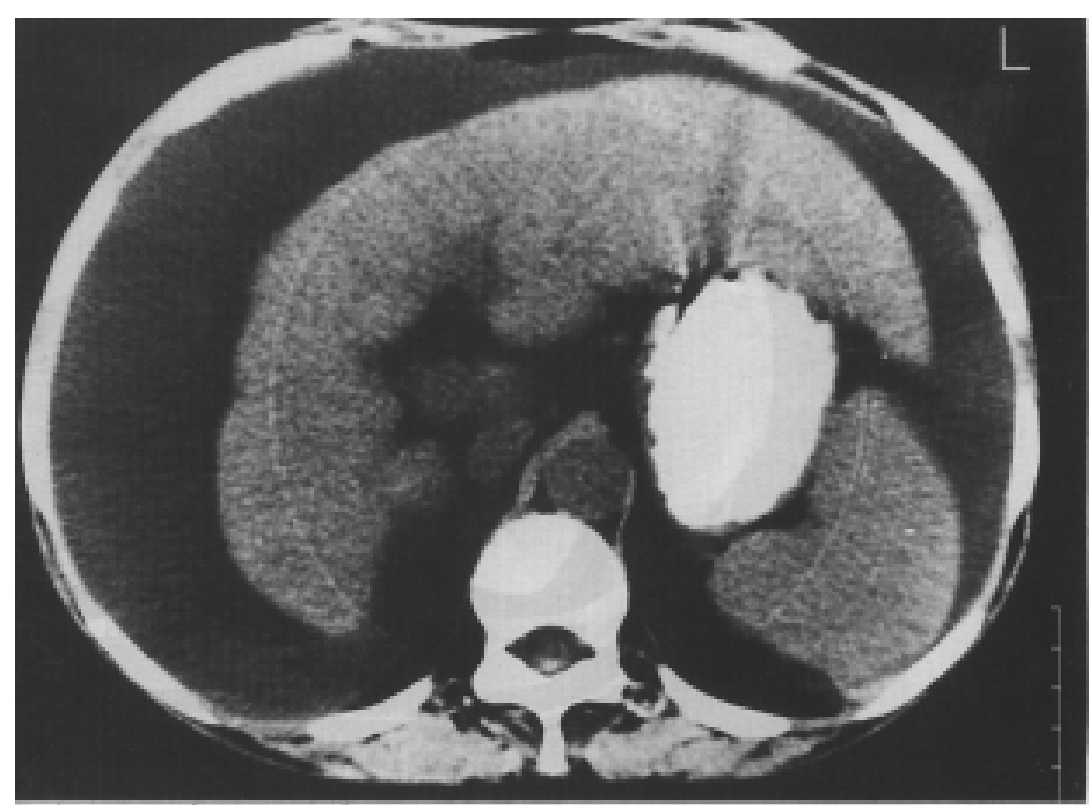

Figura 8 - Tomografia de abdome, demonstrando ascite volumosa, associada a sinais de cirrose hepática. 


\subsubsection{Pancreatite aguda}

Compreende uma entidade cuja apresentação clínica e radiológica pode assumir um espectro de variação de acordo com a severidade da doença.

Ultra-sonograficamente, os achados podem ser negativos nas formas brandas, embora se possa mostrar, nestes casos, o fator causal como coledocolitíase. A forma focal pode apresentar áreas de aumento das dimensões da glândula, hipo ou isoecóicas, sem manifestações extrapancreáticas.

Na pancreatite extensa, o pâncreas aparece difusamente aumentado e hipoecogênico em relação ao fígado, podendo estar associado à dilatação do ducto pancreático e, eventualmente, com cálculos hiperecogênicos e com sombra acústica posterior.

A tomografia computadorizada é o método radiológico de escolha para avaliação de pacientes com pancreatite em qualquer situação pois confirma o diagnóstico e quantifica o grau de acometimento da glândula.

O aumento pancreático é a primeira alteração detectável na tomografia, geralmente difuso, podendo ser segmentar em $18 \%$ dos casos, comumente na região cefálica ${ }^{(36)}$.
Nos casos mais graves, pode-se demonstrar inflamação do tecido peripancreático, espessamento da fascia pancreática e reforço heterogêneo do parênquima, após administração de contraste EV. As áreas necróticas assumem reforço menor que $50 \mathrm{UH}^{(36)}$, cuja acuracidade de detecção varia de 80 a $90 \%{ }^{(36)}$.

A ruptura do ductos pancreáticos leva à formação de coleções intra e extrapancreáticas, contendo sangue, enzimas pancreáticas e debris, podendo estar adjacentes ao pâncreas, dispersas através do peritônio, no interior de órgãos sólidos ou sítios distantes, como tórax, mediastino, pleura, pericárdio.

Coleções fluidas podem ser identificadas em 40 a $50 \%$ dos pacientes com pancreatite aguda, resolvendo espontaneamente em $50 \%$ dos $\operatorname{casos}^{(36,37)}$. Quando persistem, as coleções podem progredir para formação de pseudocistos pancreáticos, que, eventualmente, podem se infectar, progredindo para um abcesso ou apresentar sangramento. A distinção entre coleções estéreis e infectadas pode ser feita com base na demonstração de gás, no interior da coleção, sendo um achado inespecífico que ocorre em 30 a $40 \%$ dos casos, embora o diagnóstico definitivo seja feito apenas com base na aspiração do conteúdo da coleção. (Figura 9) (Tabela VII).

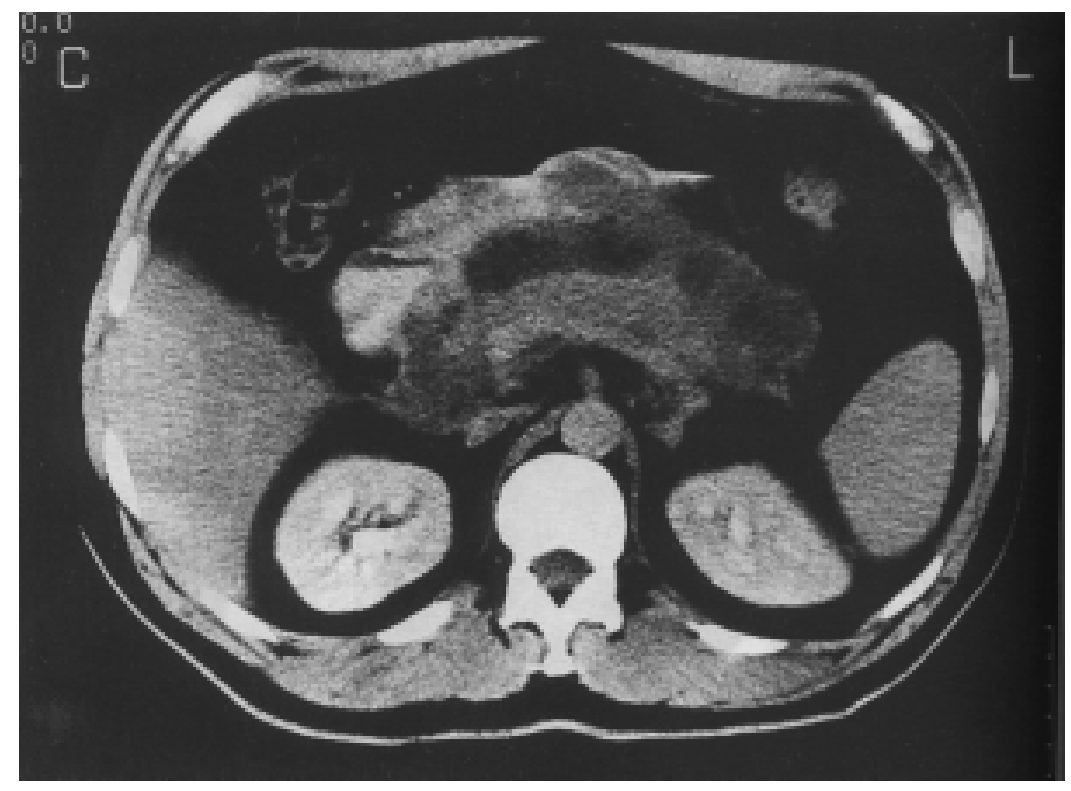

Figura 9 - Tomografia pós-contraste, demonstrando um aumento difuso do pâncreas, com áreas hipodensas por toda a glândula. 


\begin{tabular}{|c|c|c|c|c|}
\hline & & $\begin{array}{l}\text { Ordem de } \\
\text { Importância }\end{array}$ & Achados freqüentes & Limitações \\
\hline \multirow[t]{2}{*}{$\begin{array}{l}\text { Colecistite } \\
\text { Aguda }\end{array}$} & 1) & Ultra-sonografia & $\begin{array}{l}\text { - Cálculo nas vias biliares, dila- } \\
\text { tação da vesícula biliar, espes- } \\
\text { samento parietal, coleções pe- } \\
\text { ricolecísticas, sinal de Murphy } \\
\text { Ecográfico }\end{array}$ & $\begin{array}{l}\text { Obesidade, distenção gasosa intesti- } \\
\text { nal }\end{array}$ \\
\hline & 2) & Tomografia & $\begin{array}{l}\text { - Dilatacção vesicular, espessa- } \\
\text { mento parietal, coleções peri- } \\
\text { colecísticas e, eventualmente, } \\
\text { cálculos }\end{array}$ & $\begin{array}{l}\text { Não demonstra cálculos isodensos à } \\
\text { bile e o sinal de Murphy }\end{array}$ \\
\hline \multirow[t]{3}{*}{$\begin{array}{l}\text { Abcessos } \\
\text { Abdominais }\end{array}$} & 1) & Tomografia & $\begin{array}{l}\text { - Coleções com densidade variá- } \\
\text { vel, contendo ou não gás, pa- } \\
\text { redes espessadas que refor- } \\
\text { çam após contraste EV }\end{array}$ & Necessita de contraste EV e VO \\
\hline & 2) & Ultra-sonografia & $\begin{array}{l}\text { - Coleções sonoluscentes, de } \\
\text { paredes espessadas e irregula- } \\
\text { res, reforço acústico posterior, } \\
\text { níveis líquidos, debris, reverbe- } \\
\text { rações gasosas }\end{array}$ & $\begin{array}{l}\text { Obesidade, distensão gasosa intesti- } \\
\text { nal }\end{array}$ \\
\hline & 3) & Rx simples & $\begin{array}{l}\text { - Coleções gasosas extra-intesti- } \\
\text { nais deslocando alças, eleva- } \\
\text { ção da cúpula diafragmática, } \\
\text { com redução da excursão res- } \\
\text { piratória }\end{array}$ & Baixa sensibilidade e especificidade \\
\hline \multirow[t]{3}{*}{$\begin{array}{l}\text { Pancreatite } \\
\text { Aguda }\end{array}$} & 1) & Tomografia & $\begin{array}{l}\text { - Aumento das dimensões do } \\
\text { pâncreas, inflamação peripan- } \\
\text { creática, reforço heterogêneo } \\
\text { do parênquima pós-contraste } \\
\text { EV, coleções fluidas }\end{array}$ & Necessita de contraste EV e VO \\
\hline & 2) & Ultra-sonografia & $\begin{array}{l}\text { - Aumento focal ou difuso com } \\
\text { hipoecogenicidade do pân- } \\
\text { creas, coleções peripancreáti- } \\
\text { cas, coledocolitíase, dilatação } \\
\text { do ducto pancreático }\end{array}$ & $\begin{array}{l}\text { Obesidade, distensão gasosa intesti- } \\
\text { nal }\end{array}$ \\
\hline & 3) & Rx simples & $\begin{array}{l}\text { - Dilatação de alça duodenal } \\
\text { (alça sentinela) } \\
\text { - Borrando da sombra do psoas } \\
\text { à esquerda } \\
\text { - Dilatação do cólon transverso, } \\
\text { com espasmo na região da flexu- } \\
\text { ra esplênica em } 8 \% \text { dos casos. }\end{array}$ & Baixa sensibilidade e especificidade \\
\hline
\end{tabular}


LUCCHESI FR et al. The role of radiology in Intensive Care Units. Medicina, Ribeirão Preto, 31: 517-531, oct./dec. 1998.

ABSTRACT: In this critical review, we analyze the role of radiology in Intensive Care Units, discussing conventional radiology and other new imaging methods, such ultrasound and computed tomography. We included in this work the most common diseases in Intensive Care Units, focusing cardiopulmonary system and digest tract.

UNITERMS: Radiology. Thoracic Radiography. Radiography, Abdominal. Intensive Care Units.

\section{REFERÊNCIAS BIBLIOGRÁFICAS}

1 - MACMAHON H \& GIGER M. Portable chest radiography techniques and teleradiology. Radiol Clin North Am 34: 1-20, 1996.

2 - MILLER WT. The radiologist in the Intensive Care Unit. Semin Roentgenol 32: 86-88, 1997.

3 - MILLER WT. The chest radiography in the Intensive Care Unit. Semin Roentgenol 32: 89-101, 1997.

4 - BROWN K \& KALLMAN C. Imaging procedures. In: BOGARD FS \& SUE DY, eds. Current critical care diagnosis \& treatment, Appleton \& Lange, Connecticut, p.245-293, 1994

5 - CASCADE PN \& KAZEROONI EA. Aspectos da radiografia de tórax na Unidade de Terapia Intensiva. Clín Ter Intensiva 10: $251-269,1994$.

6 - WINER-MURAM HT et al. Pneumonia and ARDS in patients receiving mechanical ventilation: Diagnostic accuracy of chest radiography. Radiology 188: 479-485, 1993.

7 - HENSCHKE Cl et al. Accuracy and efficacy of chest radiography in the Intensive Care Unit. Radiol Clin North Am 34: 21-31, 1996.

8 - LIPCHIK RJ \& KUZO RS. Nosocomial pneumonia. Radiol Clin North Am 34: 47-58, 1996.

9 - MILLER Jr WT. Thoracic computed tomography in the Intensive Care Unit. Semin Roentgenol 32:117-121, 1997.

10 - PERUZZI W \& et al. Portable chest roentgenography and computed tomography in critically III patients. Chest 93: 722-726, 1988.

11 - SHIFRIN RY \& CHOPLIN RH. Aspiration in patients in critical care units. Radiol Clin North Am 34: 83-96, 1996.

12 - LANDAY MJ \& CHRISTENSEN EE \& BYNUM LJ. Pulmonary manifestation of acute aspiration of gastric contents. Am J Roentgenol 131: 587-592, 1978.

13 - LORBER B \& SWENSON RM. Bacteriology of aspiration pneumonia. Ann Intern Med 81:329-331, 1974.

14 - DESAI SR \& HANSELL DM. Lung imaging in the adult respiratory distress syndrome: current practice and new insights. Intensive Care Med 23: 7-15, 1997.
15 - RAASCH BN \& al. Pleural effusion: explanation of some typical appearances. Am J Roentgenol 139: 99-904, 1982.

16 - RUSKIN JA \& et al. Detection of pleural effusions on supine chest radiographs. Am J Roentgenol 148: 681-683, 1987.

17 - WOODRING JH. Recognition of pleural effusion on supine radiographs: How much fluid Is required? Am J Roentgenol 142: 59-64, 1984.

18 - COLLINS JD et al. Minimal detectable pleural effusions. Radiology 105: 51-53, 1972.

19 - TOCINO I \& WESTCOTT JL. Barotrauma. Radiol Clin North Am 34: 59-81, 1996.

20 - TOCINO IM; MILLER MH \& FAIRFAX WR. Distribution of pneumothorax in the supine and semirecumbent critically III adult. Am J Roentgenol 144: 901-905, 1985.

21 - GREENE R \& McLOUD TC \& STARK P. Pneumothorax. Semin Roentgenol 12: 313-325, 1977

22 - WESTCOTT JL \& ZITER FMH. Supine subpulmonary Pneumothorax. Am J Roentgenol 137: 69-701, 1981.

23 - MOSKOWITZ PS \& GRISCOM NT. The medial pneumothorax. Radiology 120:143-147, 1976.

24 - GORDON R. The deep sulcus sign. Radiology 136: 25-27, 1980.

25 - TAPSON VF. Pulmonary embolism: The diagnostic repertoire. Chest 112: 578-580, 1997.

26 - ARONCHICK JM \& MILLER WT. Tubes and lines in the intensive care setting. Semin Roentgenol 32:102-116, 1997.

27 - MARKS WM et al. Intestinal pseudotumors. A problem in computed tomography solved by direct techniques. Gastrointest Radiol 5: 155-160,1980

28-MEGIBOW AJ \& ZERHOUNI EA. Techniques of gastrintestinal computed tomography. In: MEGIBOW AJ \& BALTHAZAR EJ, eds Computed tomography of the gastrintestinal tract. CV Mosby, St.Louis, p 1-32,1986.

29 - MILLER RE. The radiological evaluation of intraperitoneal gas (pneumoperitonium). CRC Crit Rev Clin Radiol Nucl Med 4: $61-85,1973$ 
30 - MENUCK L \& SIMERS PP. Pneumoperitoneum: Importance of right upper quadrant features. Am J Roentgenol 127: 753-756, 1976.

31 - MILLER WT. The abdomen in the Intensive Care Unit. Seminars Roentgenol 32:122-127, 1997.

32 - TOMCHISK FS, WITTEMBERG J \& OTTIGER LW. Roentnographic spectrum of wel infarction. Radiology 96: 249-260, 1970.

33 - HÁ HK. CT in the early detection of strangulation in intestinal obstruction. Semin Ultrasound CT MR 16:141-150, 1995.

34 - WINTER III \& LAING FC. Hepatic ultrasound. In: MARGULIS $A R$ \& BURHENNE HJ, eds. Alimentary tract radiology. 5th. Mosby, St. Louis, p. 1475-1476, 1994.

35 - SCHNEEKLOTH G; TERRIER F \& FUCHS WA. Computed tomography of intraperitoneal abcesses. Gastrointest Radiol 7: 35-41,1982.

36 - BALTAZAR El et al. Acute pancreatitis: Prognostic value of CT. Radiology 156: 767-772, 1985.
37 - FREENE PC \& ROTORMAN CA. Inflamatory disease of the pancreas. In: FREENY PC \& STEVENSON GW, eds. Alimentary tract radiology, Mosby, St. Louis, p.1052-1090, 1993.

\section{BIBLIOGRAFIA RECOMENDADA}

1 - FEDERLE MP \& JEFFREY RB. Hemoperitoneum studied by computed tomography, Radioloy 148:187-192, 1983.

2 - INDECK M. et al. Risk and benefit of transporting ICU patient for special studies. J Trauma 28:1020-1025, 1988.

3 - NOVELLINE RA. Abdomen: Nontraumatic emergencies. In: HARRIS JH, eds. The Radiology of emergency medicine, 3th ed, Williams \& Wilkins, Baltimore, p.819-895, 1993.

Recebido para publicação em 04/03/98

Aprovado para publicação em 07/10/98 